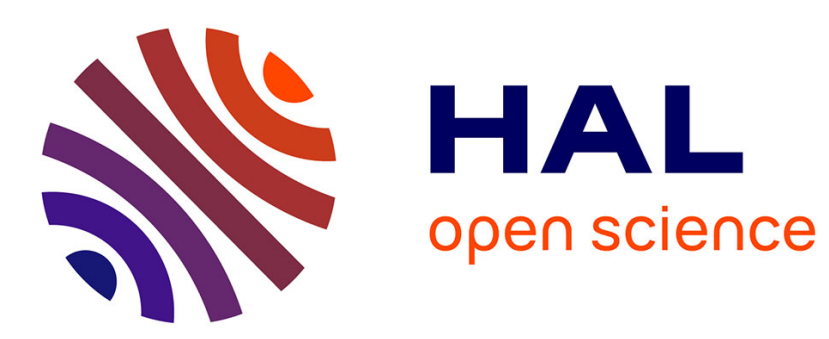

\title{
A note on Variance Bounding for continuous time Markov chains
}

Fabrizio Leisen, Cecilia Prosdocimi

\section{To cite this version:}

Fabrizio Leisen, Cecilia Prosdocimi. A note on Variance Bounding for continuous time Markov chains. Statistics and Probability Letters, 2010, 81 (1), pp.153. 10.1016/j.spl.2010.10.009 . hal-00698846

\section{HAL Id: hal-00698846 https://hal.science/hal-00698846}

Submitted on 18 May 2012

HAL is a multi-disciplinary open access archive for the deposit and dissemination of scientific research documents, whether they are published or not. The documents may come from teaching and research institutions in France or abroad, or from public or private research centers.
L'archive ouverte pluridisciplinaire HAL, est destinée au dépôt et à la diffusion de documents scientifiques de niveau recherche, publiés ou non, émanant des établissements d'enseignement et de recherche français ou étrangers, des laboratoires publics ou privés. 


\section{Accepted Manuscript}

A note on Variance Bounding for continuous time Markov chains

Fabrizio Leisen, Cecilia Prosdocimi

PII:

S0167-7152(10)00285-3

DOI:

10.1016/j.spl.2010.10.009

Reference: STAPRO 5821

To appear in: $\quad$ Statistics and Probability Letters

Received date: 22 March 2010

Revised date: 24 September 2010

Accepted date: 9 October 2010

Please cite this article as: Leisen, F., Prosdocimi, C., A note on Variance Bounding for continuous time Markov chains. Statistics and Probability Letters (2010),

doi:10.1016/j.spl.2010.10.009

This is a PDF file of an unedited manuscript that has been accepted for publication. As a service to our customers we are providing this early version of the manuscript. The manuscript will undergo copyediting, typesetting, and review of the resulting proof before it is published in its final form. Please note that during the production process errors may be discovered which could affect the content, and all legal disclaimers that apply to the journal pertain. 


\title{
A note on Variance Bounding for continuous time Markov chains
}

\author{
Fabrizio Leisen \\ Universidad Carlos III de Madrid, Spain \\ and \\ Cecilia Prosdocimi \\ DIMAD, University of Florence, Italy
}

\begin{abstract}
In this paper the notion of Variance Bounding introduced by Roberts and Rosenthal (2008) is extended to continuous time Markov Chains. Moreover, it is proven that, as in the discrete time case, the notion of Variance Bounding for reversible Markov Chains is equivalent to the existence of a central limit theorem. A connection with the continuous time Peskun ordering, introduced by Leisen and Mira (2008), concludes the paper.
\end{abstract}

Keywords: Variance Bounding, Central Limit Theorems, Peskun Ordering.

\section{Introduction}

Roberts and Rosenthal (2008) introduced the notion of Variance Bounding for discrete time Markov chains (DTMC). Let $v(h, P)$ be the asymptotic variance of a DTMC with transition kernel $P$, stationary w.r.t. a distribution $\pi$. Let $\operatorname{Var}_{\pi}(h)$ be the stationary variance. The transition kernel $P$ is Variance Bounding if there exists $K<\infty$ such that for all real valued functions $h$ we have $v(h, P) \leq K \operatorname{Var}_{\pi}(h)$. For a reversible P, Roberts et al. (2008) proved that the variance bounding of the DTMC is a necessary and sufficient condition to get a central limit theorem (CLT). In this paper, the notion of variance bounding is extended to continuous time Markov Chains and it is shown that a reversible continuous time Markov Chain (CTMC) satisfies a usual CLT if and only if it is variance bounding. It is well known that in the Markov Chain Monte Carlo (MCMC) setting we have a degree of freedom in the choice of the Markov Chain that we run in the simulation. Many criterias have been thus developed to maximize the efficiency of the MCMC simulation. One of the most notable is the Peskun ordering (see Peskun, 1973; Tierney, 1998 for discrete time), which allows to compare the asymptotic 
variances of different Markov Chains. In Roberts et al. (2008) it is shown that if a Markov Chain dominates another one in the Peskun ordering, and the latter is variance bounding then the former is variance bounding as well. Peskun ordering was extended to CTMCs by Leisen and Mira (2008). The last Section of the paper gives a connection between variance bounding in continuos time and the continuous time Peskun ordering.

\section{Preliminaries}

Let $\left(X_{t}\right)_{t \in \mathbb{R}^{+}}$be a continuous time Markov Chain (CTMC) with values in a measurable space $(\mathcal{X}, \mathcal{F})$ and with generator $G$. Throughout the paper we assume that the CTMC is reversible with respect to $\pi$, i.e.

$\int_{x \in A} \pi(d x) P^{t}(x, B)=\int_{x \in B} \pi(d x) P^{t}(x, A) \quad$ for any $A, B \in \mathcal{F} \quad$ and $t \in \mathbb{R}^{+}$, where $P^{t}(x, d y)$ is the $t$-step transition kernel.

Definition 1. (see Bhattacharya (1982)) For every $h: \mathcal{X} \rightarrow \mathbb{R}$, the asymptotic variance of the CTMC $\left(X_{t}\right)_{t \in \mathbb{R}^{+}}$is defined as

$$
v(h, G):=\lim _{n \rightarrow+\infty} \frac{1}{n} \operatorname{Var}\left(\int_{0}^{n t} h\left(X_{s}\right) d s\right) .
$$

Bhattacharya (1982) shows that a CLT holds true for functions $h: \mathcal{X} \rightarrow$ $\mathbb{R}$ in the range of the generator $G$. In the paper, we thus will refer to functions $h$ in this class. In particular for functions $h$ in the range of the generator, we have

$$
v(h, G)=-2\left\langle h, G^{-1} h\right\rangle,
$$

where $\langle f, g\rangle=\int f(x) g(x) \pi(x)$ denotes the scalar product in $L^{2}(\pi)$. Suppose that the chain starts in stationarity and that $\operatorname{Var}_{\pi}(h)=E\left[\left(h\left(X_{0}\right)-\right.\right.$ $\left.\left.E\left[h\left(X_{0}\right)\right]\right)^{2}\right]$ is the stationary variance. Then, the natural extension of the definition of variance bounding to CTMC is the following

Definition 2. The generator $G$ is Variance Bounding if there exists $K<\infty$ such that for all $h$ in the range of the generator we have

$$
v(h, G) \leq K \operatorname{Var}_{\pi}(h) .
$$

Let $\sigma(P)$ be the spectra of an operator $P$. In particular, the spectra of a generator $G$ is a subset of $\mathbb{R}^{-}$. We denote by $E_{P}$ the resolution of the 
identity of an operator $P$ given in the spectral theorem (see Conway, 1985; Rosenthal 2003; Mira and Leisen, 2009). We thus have

$$
g(P)=\int_{\sigma(P)} g(\lambda) E_{P}(d \lambda)
$$

for every bounded and measurable function $g: \sigma(P) \rightarrow \mathbb{R}$. Moreover, given a bounded and measurable function $g$, let $E_{g, P}$ be the spectral measure associated with $g$ and $P$, so that $E_{g, P}(A)=\left\langle g, E_{P}(A) g\right\rangle$ and

$$
\langle g, h(P) g\rangle=\int_{\sigma(P)} h(\lambda) E_{g, P}(d \lambda)
$$

for every bounded and measurable function $h: \mathbb{R} \rightarrow \mathbb{R}$. Let $G$ be the generator of a CTMC. Let

$$
\Lambda=\Lambda(G):=\sup _{\lambda \in \sigma(G)} \lambda
$$

Theorem 1. Let $G$ be the generator of a CTMC reversible w.r.t. $\pi$. $G$ is variance bounding if and only if $\Lambda<0$.

Proof. We prove the sufficiency part of the theorem by contradiction. Thus suppose that $\sup _{\lambda \in \sigma(G)} \lambda=0$ and let $r<0$. Then $E_{G}((r, 0])$ is non zero, so there exists $h \in L_{0}^{2}(\pi)$ in the range of $E_{G}((r, 0])$ such that

$$
\begin{aligned}
v(h, G) & =-2\left\langle h, G^{-1} h\right\rangle=-\int_{\sigma(G)} \frac{2}{\lambda} E_{h, G}(d \lambda) \geq-\int_{r}^{0} \frac{2}{\lambda} E_{h, G}(d \lambda) \\
& \geq \frac{-2}{r} E_{h, G}((r, 0])=\frac{-2}{r}\left\langle h, E_{G}((r, 0]) h\right\rangle=\frac{-2}{r}\langle h, h\rangle=-\frac{2}{r} \operatorname{Var}_{\pi}(h) .
\end{aligned}
$$

Hence

$$
\frac{v(h, G)}{\operatorname{Var}_{\pi}(h)} \geq-\frac{2}{r}
$$

and

$$
\sup _{h \in L_{0}^{2}(\pi)} \frac{v(h, G)}{\operatorname{Var}_{\pi}(h)} \geq \sup _{r<0}-\frac{2}{r}=+\infty .
$$

Thus $G$ is not variance bounding, and we get a contradiction. Hence $\Lambda=$ $\Lambda(G)=\sup _{\lambda \in \sigma(G)} \lambda<0$. 
Conversely, suppose that $\Lambda=\Lambda(G)=\sup _{\lambda \in \sigma(G)} \lambda<0$. Hence for $h \in L_{0}^{2}(\pi)$ in the range of the generator

$$
\begin{aligned}
v(h, G) & =-2\left\langle h, G^{-1} h\right\rangle=-\int_{\sigma(G)} \frac{2}{\lambda} E_{h, G}(d \lambda) \leq-\frac{2}{\Lambda} \int_{\sigma(G)}\left\langle h, E_{G}(d \lambda) h\right\rangle \\
& =-\frac{2}{\Lambda}\langle h, h\rangle=-\frac{2}{\Lambda} \operatorname{Var}_{\pi}(h) .
\end{aligned}
$$

Thus $G$ is variance bounding with $K=-\frac{2}{\Lambda}$.

\section{Relation with central limit theorems}

The following theorem shows that for reversible CTMC, the hypothesis of variance bounding is a necessary and sufficient condition to get a usual CLT. A similar result holds for DTMC (see Roberts et al. (2008)).

Theorem 2. Let $G$ be reversible w.r.t. $\pi . G$ is variance bounding if and only if every $h \in L^{2}(\pi)$ in the range of the generator $G$ satisfies a usual CLT for $G$.

Proof. If $G$ is variance bounding, then by Theorem 1 we have $\Lambda=\Lambda(G)=$ $\sup \lambda<0$. Hence, $\lambda \in \sigma(G)$

$$
v(h, G) \leq-\frac{2}{\Lambda}\langle h, h\rangle<+\infty
$$

and a CLT holds (see Bhattacharya, 1982; Kipnis and Varadhan, 1986).

Conversely, suppose that $G$ satisfies a CLT for every $h \in L^{2}(\pi)$ in the range of $G$, but $G$ is not variance bounding. Then by Theorem 1 we have $\Lambda=$ $\Lambda(G)=\sup _{\lambda \in \sigma(G)} \lambda=0$. Thus $E_{G}((r, 0]) \neq 0$ for any $r<0$, and there must exist infinitely many $m \in \mathbb{N}$ such that $E_{G}\left(\left(-2^{-m},-2^{-m-1}\right]\right)$ is non zero. Let $m_{1}<m_{2}<\ldots$ (and thus $\left.m_{i} \geq i\right)$ such that $E_{G}\left(\left(-2^{-m_{i}},-2^{-m_{i}-1}\right]\right)$ is non zero. Let $g_{i}$ in the range of $E_{G}\left(\left(-2^{-m_{i}},-2^{-m_{i}-1}\right]\right)$ with $\left\|g_{i}\right\|=\left\langle g_{i}, g_{i}\right\rangle=1$. The $g_{i}$ are thus orthonormal and furthermore

$$
\begin{aligned}
\left\langle g_{i}, G^{-1} g_{i}\right\rangle & =\int_{\sigma(G)} \lambda^{-1} E_{g_{i}, G}(d \lambda)=\int_{-2^{-m_{i}}}^{-2^{-m_{i}-1}} \lambda^{-1} E_{g_{i}, G}(d \lambda) \\
& \leq-2^{m_{i}} \int_{-2^{-m_{i}}}^{-2^{-m_{i}-1}} E_{g_{i}, G}(d \lambda)=-2^{m_{i}}<g_{i}, g_{i}>=-2^{m_{i}} .
\end{aligned}
$$


Define $h=\sum_{i=1}^{+\infty} 2^{-\frac{i}{2}} g_{i}$. From the orthonormality

$\operatorname{Var}_{\pi}(h)=\langle h, h\rangle=\left\langle\sum_{i=1}^{+\infty} 2^{-\frac{i}{2}} g_{i}, \sum_{i=1}^{+\infty} 2^{-\frac{i}{2}} g_{i}\right\rangle=\sum_{i=1}^{+\infty}\left(2^{-\frac{i}{2}}\right)^{2}\left\langle g_{i}, g_{i}\right\rangle=\sum_{i=1}^{+\infty} 2^{-i}=1$.

On the other hand, since $\left\langle g_{i}, G^{-1} g_{i}\right\rangle \leq-2^{m_{i}}$ and $\left\langle g_{i}, G^{-1} g_{j}\right\rangle=0$ for all $i \neq j$

$$
\begin{aligned}
v(h, G) & =-2\left\langle h, G^{-1} h\right\rangle=-2\left\langle\sum_{i=1}^{+\infty} 2^{-\frac{i}{2}} g_{i}, G^{-1} \sum_{i=1}^{+\infty} 2^{-\frac{i}{2}} g_{i}\right\rangle=-2 \sum_{i=1}^{+\infty}\left(2^{-\frac{i}{2}}\right)^{2}\left\langle g_{i}, G^{-1} g_{i}\right\rangle \\
& \geq-2 \sum_{i=1}^{+\infty} 2^{-i}\left(-2^{m_{i}}\right)=2 \sum_{i=1}^{+\infty} 2^{-i}\left(2^{m_{i}}\right) \geq 2 \sum_{i=1}^{+\infty} 2^{-i} 2^{i}=+\infty .
\end{aligned}
$$

Thus $G$ does not satisfy a CLT, but this is in contrast with the hypothesis. Hence $G$ is variance bounding.

\section{Relation with Peskun ordering}

In Leisen and Mira (2008), the Peskun ordering has been extended to CTMC. For MC with finite or countable state space, a natural definition for continuous time Peskun ordering is the following

Definition 3. Suppose that $G_{1}=(g(1))_{i j}$ and $G_{2}=(g(2))_{i j}$ are the generators of two CTMCs, both stationary with respect to a distribution $\pi$ (i.e., $\pi G_{1}=0, \pi G_{2}=0$ ), and suppose that the two chains are uniformizables. Then we say that $G_{1}$ dominates $G_{2}$ in the continuous time Peskun ordering and we write $G_{1} \succeq_{E P} G_{2}$ if

$$
g(1)_{i j} \geq g(2)_{i j}, \quad \forall i \neq j .
$$

For general state space, we have to consider generators $G$ which can be written as

$$
G f(x)=\int f(y) Q(x, d y)
$$

where the kernel $Q$ is defined in terms of the transition kernel $P$ as

$$
Q(x, d y)=\frac{\partial}{\partial t} P(t, x, d y)_{\mid t=0}
$$

In this case the Peskun ordering in a general state space $(\mathcal{X}, \mathcal{F})$ is defined in the following way 
Definition 4. Let $G_{1}$ and $G_{2}$ be the generators of two CTMCs admitting the representation (1) with kernels $Q_{1}$ and $Q_{2}$ respectively, both stationary with respect to a common distribution $\pi$ on $\mathcal{X}$. Assume $\sup _{x} Q_{i}(x, \mathcal{X} \backslash\{x\})<\infty$, for $i=1,2$. Then $G_{1}$ dominates $G_{2}$ in the continuous time Peskun ordering, $G_{1} \succeq_{E P} G_{2}$, if

$$
Q_{1}(x, A \backslash\{x\}) \geq Q_{2}(x, A \backslash\{x\}), \quad \forall A \in \mathcal{F} .
$$

In Leisen et al. (2008) the following theorem for continuous time Peskun ordering is proven

Theorem 3. If $G_{1} \succeq_{E P} G_{2}$ and if the corresponding CTMCs are reversible, then $G_{2}-G_{1}$ is a positive operator.

As a consequence of Theorem 3, the following theorem holds.

Theorem 4. Let $G_{1}, G_{2}$ be the generators of two CTMCs reversible w.r.t. $\pi$, which admit a representation as in equation (1). Let $G_{1} \succeq_{E P} G_{2}$, and let $G_{2}$ be variance bounding. Then $G_{1}$ is variance bounding.

Proof. By Theorem 3, the operator $G_{2}-G_{1}$ is positive, thus $\sup \sigma\left(G_{2}\right) \geq$ $\sup \sigma\left(G_{1}\right)$. Applying twice Theorem 1 the result easily follows.

\section{Acknowledgements}

The authors are grateful to the two anonymous referees for the many detailed suggestions which led to significant improvement in the presentation of the paper. Moreover, the authors would like to thank Prof. Paolo Dai Pra for the helpful discussions.

\section{References}

Bhattacharya R., On the functional central limit theorem and the law of the iterated logarithm for Markov processes, Z. Wahrsch. Verw. Gebiete, 60(2): 185-201, 1982.

Conway J. B., A course in Functionals Analysis, Springer Verlag, 1985.

Kipnis C, and Varadhan R.S. Central Limit Theorem for Additive Functionals of Reversible Markov Process and Applications to Simple Exclusions, Commun. Math. Phys., 104: 1-19, 1986.

Leisen F. and Mira A., An extension of Peskun and Tierney orderings to continuous time Markov chains, Statistica Sinica, 18: 1641-1651, 2008. 
Mira A. and Leisen F., Covariance Ordering for discrete and continuous time Markov Chains, Statistica Sinica, 19: 651-666, 2009.

Peskun P.H., Optimum Monte Carlo sampling using Markov Chains, Biometrika, 60: 607-612, 1973.

Rosenthal J. S., Asymptotic Variance and convergence rates of nearly periodic MCMC algorithm, JASA, 98: 169-177, 2003.

Roberts G. O. and Rosenthal J. S., Geometric ergodicity and hybrid Markov chains, Electronic Comm. Prob., 2: 13-25, 1997.

Roberts G. O. and Rosenthal J. S., Variance Bounding Markov Chains, Ann. of App. Prob., 18: 1201-1214, 2008.

Tierney L., A Note on Metropolis Hastings Kernels for General state spaces, Ann. of App. Prob., 8: 1-9, 1998. 